\title{
The superimposition of heat-induced chiasma frequency changes in Locusta migratoria
}

\author{
S. A. Henderson and \\ M. E. Buss*
}

Department of Genetics, University of Cambridge, Cambridge, U.K.

Inbred laboratory stocks of the locust Locusta migratoria were used in two different types of experiment involving the superimposition of heat-induced changes in chiasma frequency. The first involved the administration of two heattreatments at $40^{\circ} \mathrm{C}$ alternating with a return to $30^{\circ} \mathrm{C}$. This was designed to superimpose two small changes, which have been called Effect 1 (increase) and Effect 2 (decrease) upon a single larger Effect 4 (increase). Unlike the response previously reported in a similar experiment carried out with Schistocerca gregaria, an intermediate response was found here in Locusta, with recognisable Effects 1 and 2 components being superimposed upon a reduced Effect 4 increase. The aim of the second experiment was to determine the effects of chiasma frequency of an alternating programme of one day at $40^{\circ} \mathrm{C}$, followed by a return to $30^{\circ} \mathrm{C}$, maintained for a period of several weeks. This would continually superimpose small effects of all types upon one another. It was found that, rather than falling, as might have been expected, chiasma frequencies rose substantially after the first five days and remained well above control levels for the remainder of the experiment. This readily induced increase in recombination frequencies could be of practical application to those breeders interested in the production of new varieties by conventional selection.

\section{INTRODUCTION}

When locusts are heat-treated at $40^{\circ} \mathrm{C}$, four different effects on chiasma frequency can be obtained: two types of increase and two types of decrease. Following labelling with $\mathrm{H}$-thymidine to delimit the S-phase, it can be shown that three of the effects involve heat-treating different meiotic prophase stages, while the fourth, an increase, involves heat-treating spermatogonia/G1 stages (Henderson, 1970; 1988 $a$ ). They are demonstrated most successfully when relatively short heat-treatments of two to six days are given, followed by incubation at the lower, control temperature of $30^{\circ} \mathrm{C}$. These four effects were first discovered using inbred laboratory stocks of the locust Schistocerca gregaria. A second series of experiments using inbred laboratory stocks of Locusta migratoria revealed that all four effects could also be produced in this species, but there were some important differences (Buss, 1971; Buss and Henderson, 1988). These involved such things as the timing and magnitude of the effects, the very different

* Mrs. M. E. Williams. response within the chromosome size groups present, and the amount of recovery possible at a constant $30^{\circ} \mathrm{C}$.

In order to determine more about the nature of the four effects produced in Schistocerca a further question was raised: what interactions would occur between them if they were to be superimposed? Using experimental designs in which two $40^{\circ} \mathrm{C}$ heat-treatments of suitable duration were separated by an appropriate interval at $30^{\circ} \mathrm{C}$, two such experiments were carried out: (a) in one, Effect 3 was superimposed upon Effect 4 (b) in the other, Effects 1 and 2 were superimposed upon Effect 4 . The results showed clearly that, in this species, the reductions brought about by Effects 2 and 3 were both dominant to the Effect 4 increase (Henderson, 1988b).

Because there were differences between Schistocerca and Locusta in the timing, magnitude, and nature of the four effects produced by a single heat-treatment, one might also expect differences between the two species when superimposing heatinduced changes. It might be suspected from the previous work, for example, that the Effect 4 increase in Locusta might be more "dominant" 
than that produced in Schistocerca. To test this an experiment was carried out superimposing Effects 1 and 2 with Effect 4 . These are smaller effects and less likely to swamp Effect 4 than the massively dominant Effect 3 increase.

In addition to carrying out this experiment involving a single superimposition, using two heattreatments, the work was extended further by asking the following question: what would happen by repeatedly superimposing small effects upon one another? In all of the double heat-treatment experiments periods of two to four days at $40^{\circ} \mathrm{C}$ were chosen as the optimum times necessary to produce all four effects clearly. Heat-treatment for only one day at $40^{\circ} \mathrm{C}$ does produce some effects, but these tend to be small, with virtually no Effect 3 reduction (Henderson, 1988a; Buss, 1971). It could be that one day at $40^{\circ} \mathrm{C}$ is approaching the minimum period of time which can produce any effect at all. Alternatively, more potential "damage" may be produced than seen, because restitution, or recovery, may take place when locusts are quickly returned to the lower temperature.

To determine what capacity the system has for restitution, or recovery, and what the cumulative effects would be of a series of one day heat-shocks, an experiment was carried out involving the heattreatment of Locusta for one day at $40^{\circ} \mathrm{C}$, followed by a return to $30^{\circ} \mathrm{C}$, repeated in an alternating fashion for several weeks. An experiment of this type is of particular interest because it can be thought of in two different ways: either (a) the cumulative effects of one day treatments, or (b) the effect of fractionating a longer period of heattreatment into one day periods.

\section{MATERIALS AND METHODS}

The inbred stocks of $L$. migratoria used were those which were used in the earlier publications in this series (Buss and Henderson, 1988). The experimental procedure was also similar to that used before. Locusts were heat-treated in an incubator at $40^{\circ} \mathrm{C}$ followed by transfer to a second incubator maintained at a temperature of $30^{\circ} \mathrm{C}$. Two individuals were sampled on most days but, when Effect 1 was expected, more heavy sampling of two or three individuals at shorter intervals was carried out.

Testes were cleaned of fat body in insect saline and fixed in acetic-alcohol $(1: 3)$. Twenty cells were scored from each individual in propionic-orcein squash preparations. Means, sample standard deviations and standard errors were calculated.

\section{RESULTS}

\section{Control chiasma frequencies}

At the start of these experiments it was felt important to provide a new control series, to run alongside these heat-treatment experiments, to establish what chiasma frequency levels were present in individuals from this generation, in case they differed from those of previous control series. The results obtained when 29 individuals were maintained at a constant $30^{\circ} \mathrm{C}$ are provided in fig. $1(a, b)$. The curve shows low between-individual variation each day sampled, with chiasma frequencies oscillating only a little above and below a control mean of circa 14 chiasmata per cell. No reduction in chiasma frequency with age was found in this material, in contrast to the response found in the first control curves obtained (Buss and Henderson, 1988).

\section{The superimposition of Effects 1 and 2 with Effect 4}

Because of the accelerating effects of increased temperature on meiotic rate the experimental design must be chosen carefully. The second heattreatment, in addition to producing its own effects, will also speed up the passage of those cells showing the first effect through meiosis. The principles underlying such experimental design have been discussed in detail in the previous publication involving superimposition (Henderson, 1988b), and need not be relisted here.

In order to show what changes would be produced on Effect 4 by the superimposition of Effects 1 and 2, a double experiment was carried out. Thirty-eight locusts were placed in an incubator maintained at $40^{\circ} \mathrm{C}$ for four days and then all returned to $30^{\circ} \mathrm{C}$. At the end of a further two days the locusts were split into two separate experiments: (a) 18 were left at $30^{\circ} \mathrm{C}$ and sampled each day to reveal the nature of the original Effect 4 increase, i.e. providing an Effect 4 "control" series. (b) the remaining 20 were replaced at $40^{\circ} \mathrm{C}$ for a further four days, before returning them to $30^{\circ} \mathrm{C}$. These were sampled intensely around day seven, when Effect 1 was predicted, and at daily intervals thereafter.

\section{(a) The results of a single heat-treatment}

The chiasma frequencies of the individuals samples between days 6 and 13 are shown in fig. $2(a, b)$. It will be seen that, following the massive 


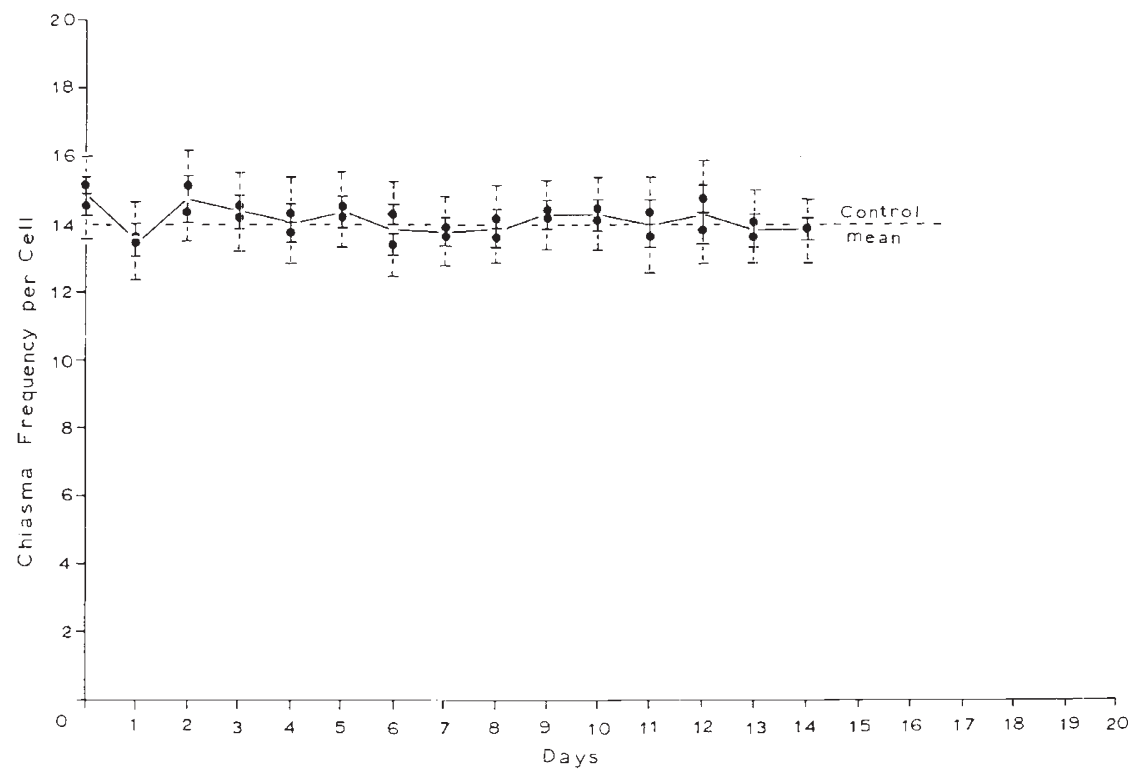

Figure 1a The chiasma frequencies obtained when control individuals were maintained at a constant $30^{\circ} \mathrm{C}$. Means, sample standard deviations and standard errors are provided for the 20 cells scored from the two individuals samples each day.

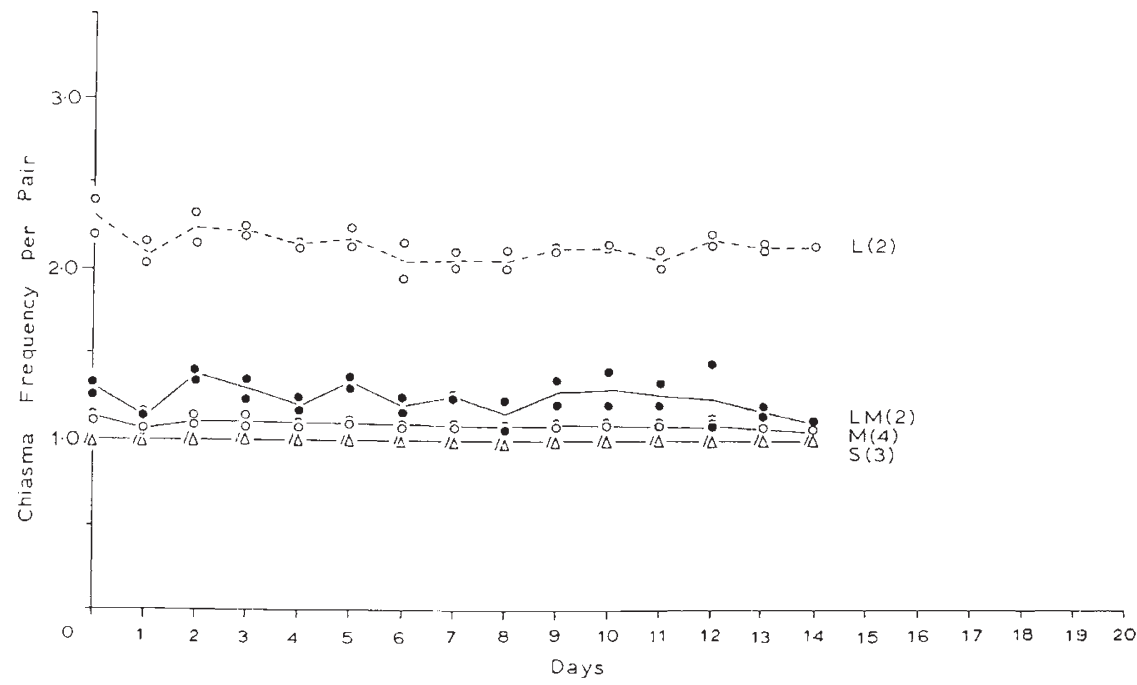

Figure 1b Chiasma frequency curves for the three size groups recognised within the complement.

Effect 3 decrease to almost total asynapsis, still being shown on day six, there was a rapid recovery on day seven, followed by a substantial and prolonged Effect 4 increase. This maintained chiasma frequencies significantly above control levels for several days. Normal levels were regained on day 13. At the peak of Effect 4 , on day 11, chiasma frequencies were so high that values as high as 21 were found in some cells. This raised the overall mean of both individuals sampled to a value of $17 \cdot 73$, compared with the control mean of circa 14

\section{(b) The effects of two combined heat-treatments}

Calculations on the accelerating effects of the higher temperature on meiotic rate $\left(Q_{10}=\right.$ circa $2-2 \cdot 3$ ) indicated that the optimum time interval between heat-treatments to superimpose the second Effects 1 and 2 with the first Effect 4, would 


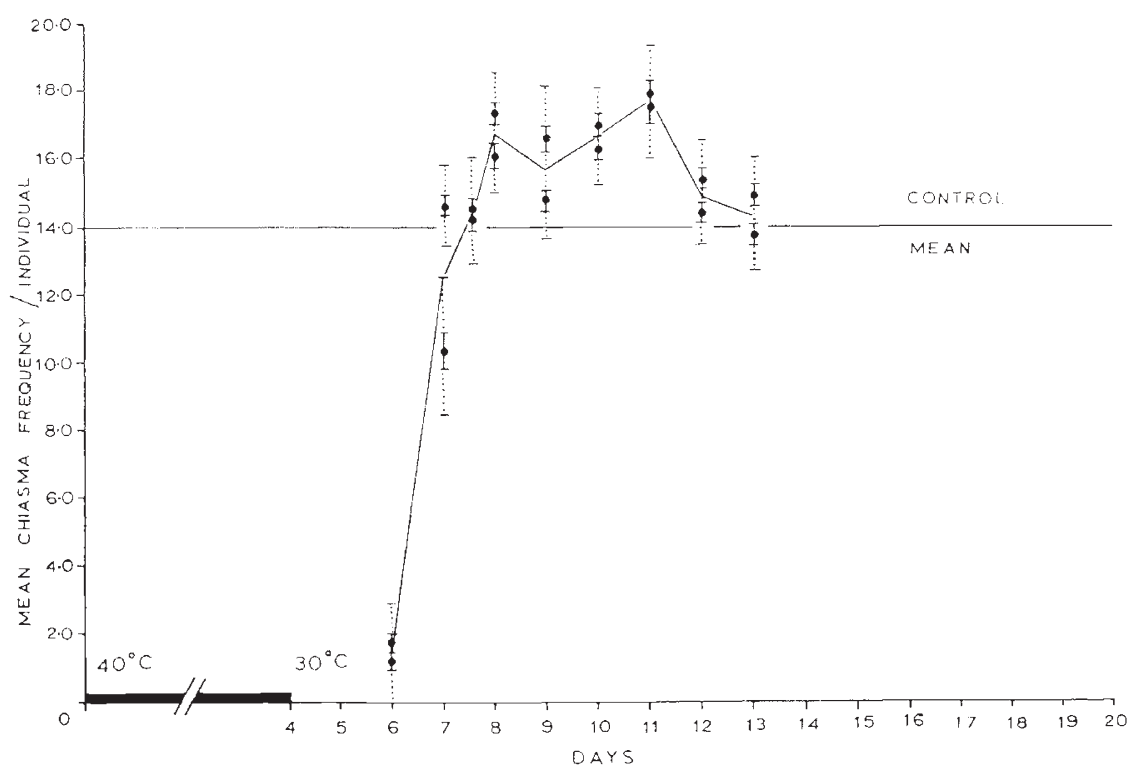

Figure 2a Chiasma frequency response with time following heat-treatment at $40^{\circ} \mathrm{C}$ for four days, followed by a return to $30^{\circ} \mathrm{C}$. Means, sample standard deviations and standard errors of 20 cells scored from each of the individuals sampled. This provides a "control" experiment for comparison with Fig. 3a.

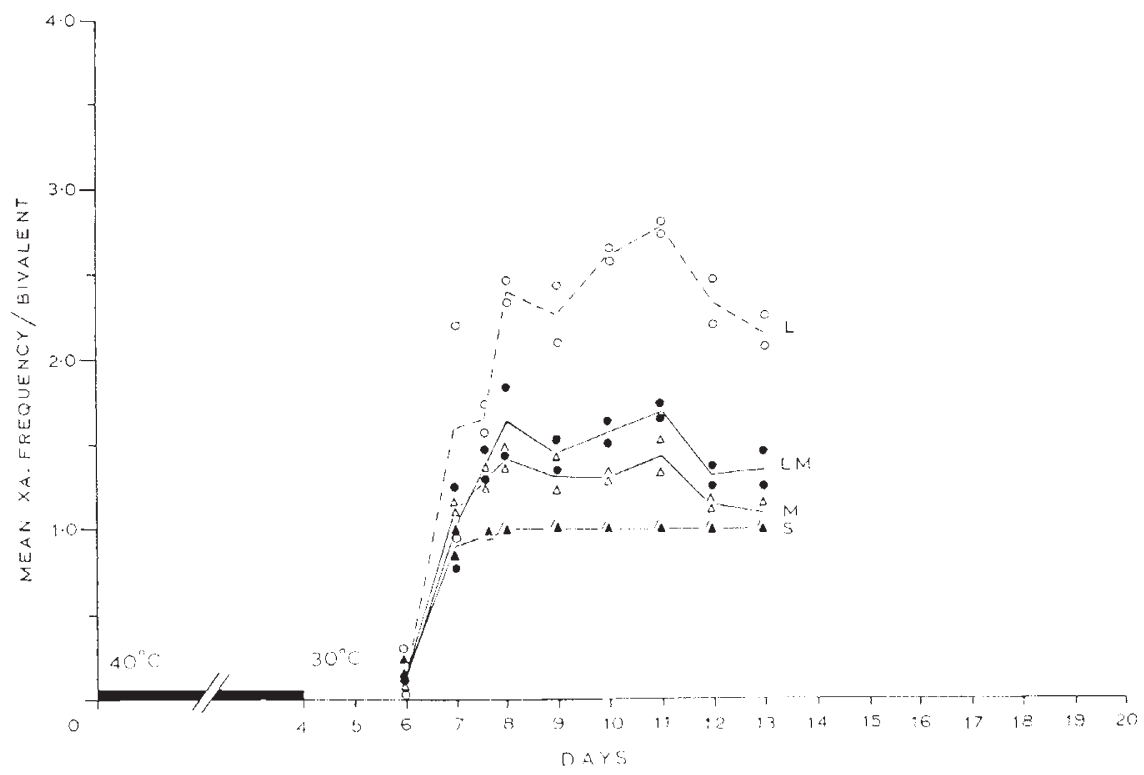

Figure $\mathbf{2 b}$ The response within the three size groups recognised in the experiment illustrated in fig. $2(\mathbf{a})$.

be two days at $30^{\circ} \mathrm{C}$. It was also calculated that any Effect 1 increase whould occur about day seven, so sampling was increased at this time. The results of superimposing a second heat-treatment of four days at $40^{\circ} \mathrm{C}$ on the original response is shown in fig. 3(a), b). In this figure the day axis is roughly "corrected" for the effects of high temperature by doubling the length of each day drawn at $40^{\circ} \mathrm{C}$. This also serves the function of helping to clarify the nature of the response in the period of crowded sampling.

Following the Effect 3 decrease, which ended 


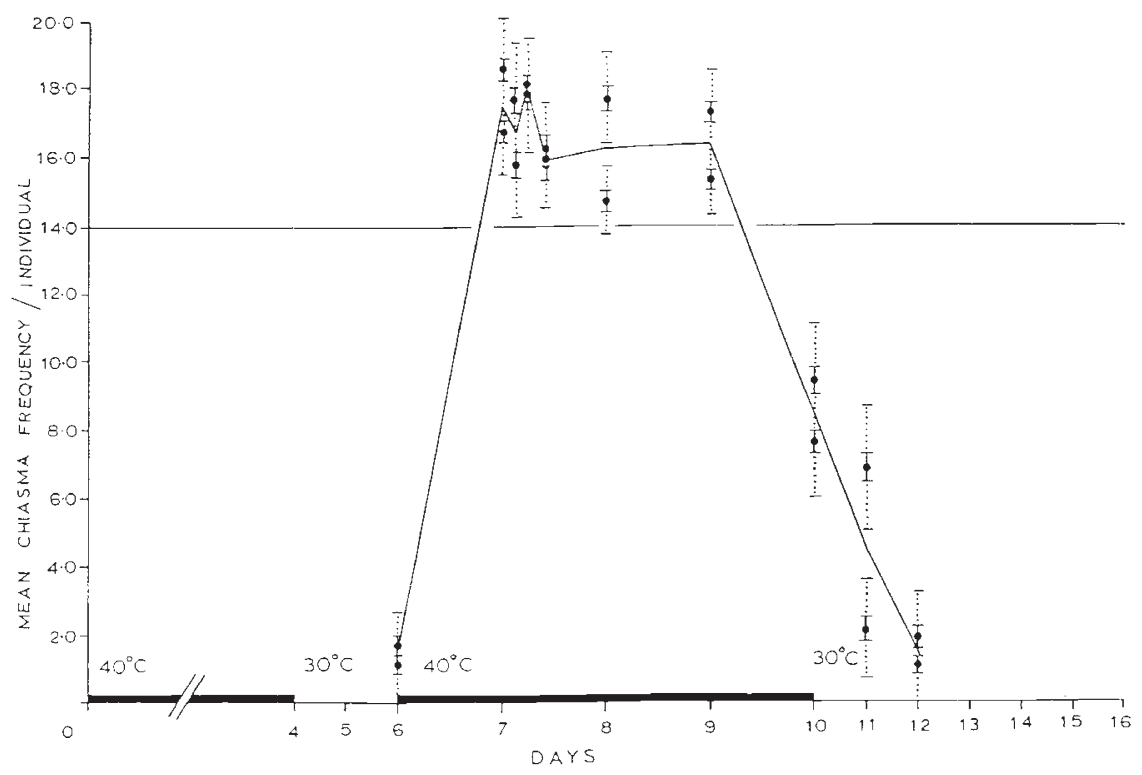

Figure 3a Chiasma frequency response with time following the superimposition of two heat-treatments of four days at $40^{\circ} \mathrm{C}$, separated by two days at $30^{\circ} \mathrm{C}$. Means, sample standard deviations and standard errors of 20 cells scored from each of the individuals sampled. The axis is extended to compensate for the doubling of the rate of cell division at $40^{\circ} \mathrm{C}$ and clarify the nature of the response when Effects 1 and 2 are superimposed on Effect 4.

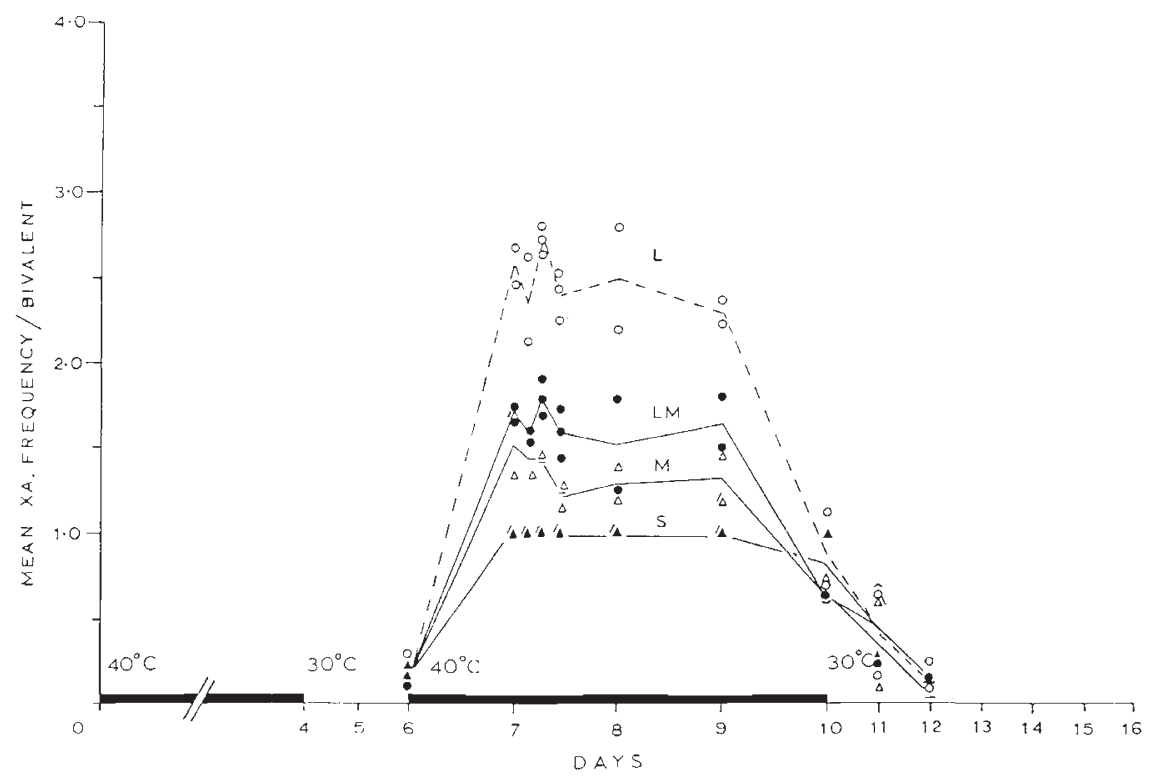

Figure 3b The response within the three size groups recognised in the experiment illustrated in fig. 3(a).

on day six, there was a rapid recovery to chiasma frequencies well above control levels on day seven, where a peak was found, corresponding to Effects 1 and 4 combined. On days eight and nine chiasma frequencies were lower than on day seven, but still above control values. The shape of the curve on these three days clearly reflected an intermediate response, with the shapes of Effects 1 and 2 superimposed on an Effect 4 increase. Following this period of increase, on days $10-12$, there was a profound drop in chiasma frequencies to a low level, in the second Effect 3 decrease. 


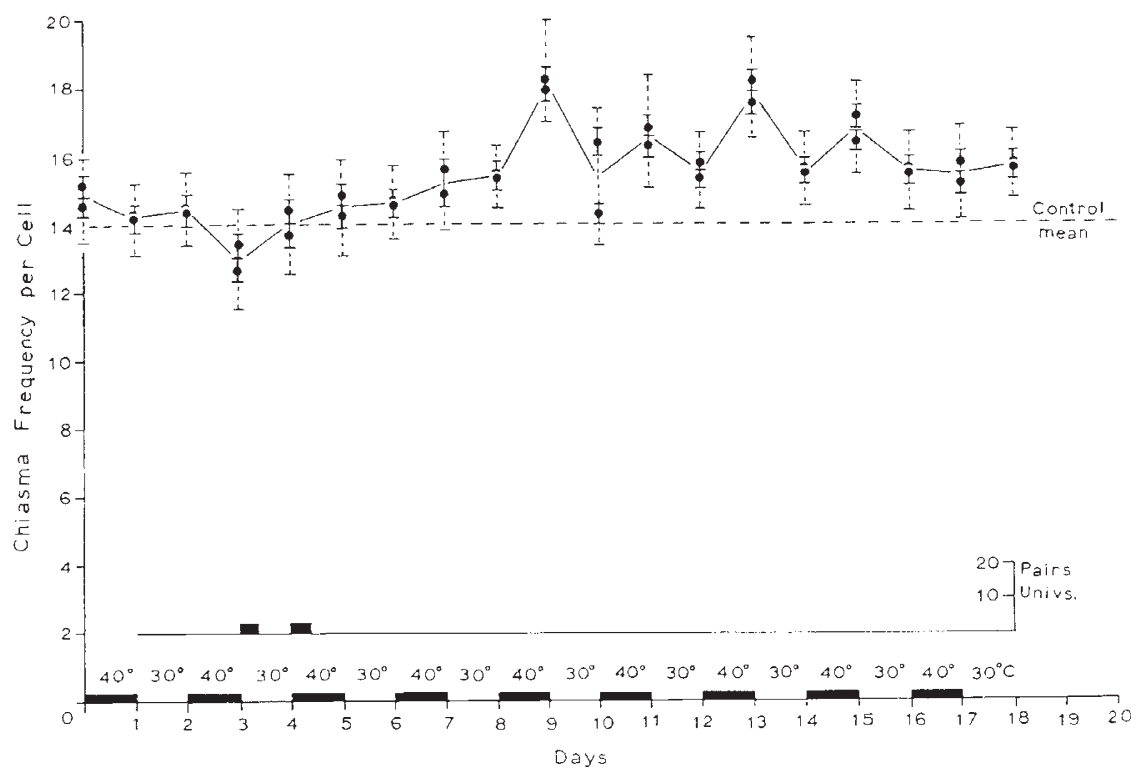

Figure 4a Chiasma frequency response obtained following an alternating daily heat-treatment of $40^{\circ} \mathrm{C}$, followed by return to $30^{\circ} \mathrm{C}$. Means, sample standard deviations and standard errors again provided for the 20 cells scored from the two individuals samples per day.

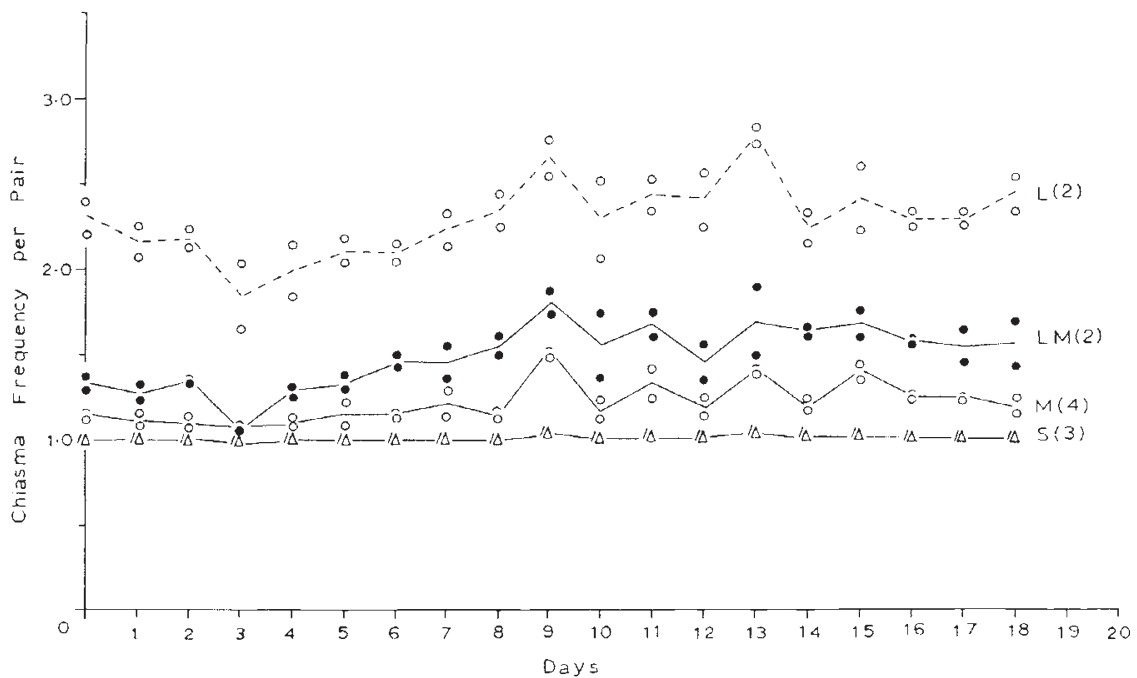

Figure $4 \mathbf{b}$ The response shown by the three size groups recognised in the experiment illustrated in fig. 4(a).

The effects of a regularly alternating one day heat-treatment

The results obtained when 36 individuals were subjected to a regularly alternating regime of one day at $40^{\circ} \mathrm{C}$ followed by one day at $30^{\circ} \mathrm{C}$ are shown in fig. (4a, b). Once again, between-individual variation each day was extremely low, which served to improve the clarity with which the response was illustrated. Although an initial Effect 1 was not seen (nor expected with daily sampling), a small Effect 2 was obtained on day three, with a few univalents being produced. A rapid return to control frequencies, with no suggestion of an Effect 3 decrease, was followed by a steady increase in chiasma frequencies over the next few days. 
Chiasma frequencies reached an Effect 4 peak of circa 18 per cell (mean) on day nine and continued well above control levels for the duration of the experiment. There was every indication that this prolonged Effect 4 increase would continue as long as the alternating heat-treatment continued, with no tendency for levels to drop below the control mean.

An analysis of the contributions made by the different size groups within the complement (fig. $4 b$ ) showed that the prolonged increase in chiasma frequencies affected all of the chromosomes within the complement. Even the smalls occasionally formed two chiasmata (unheard of in control cells), though only rarely.

\section{DISCUSSION}

The results of these and the earlier experiments show two things: (a) the four effects of heat-treatment on chiasma frequency reflect changes in chromosomal activities which are sufficiently fundamental to be produced in two unrelated locust species, $S$. gregaria and $L$. migratoria. (b) important differences exist between the two species in practice, both in the nature of the primary effects shown and in the interactions which occur when heat-induced effects are combined. These must reflect basic genetic and biochemical differences between the species.

In the case of $S$. gregaria it was found that when Effects 1 and 2 were superimposed on Effect 4, no Effect 4 increase was obtained. While a peak in the curve indicated the position of Effects 1 and 2 , chiasma frequencies stayed at, or below, control levels (Henderson, 1988b). Here, in L. migratoria, on the other hand, while a modest Effect 4 increase above control levels was obtained, the shape of the curve was noticeably changed from its original appearance to one showing an intermediate response, involving recognisable Effect 1 and 2 components. Clearly the biochemical events involved in the potential for an increased number of chiasmata to form during Effect 4 are more resistant to change in Locusta than they are in Schistocerca.

The results of the second experiment are of particular interest in revealing that there is a cumulative effect of alternating heat-treatments and that this does not lead to a reduction in chiasma frequencies, with an Effect 3 fall, as might have been expected, but an increase in chiasma frequencies, with a prolonged Effect 4 increase. This is of interest in attempting to understand the mechanisms underlying the heat-induced changes during the production of the 4 effects. It is clear that the period of time, during which Effect 3 is induced, must be much longer than one day at $40^{\circ} \mathrm{C}$ and is not severely affected by a one day heat-treatment. Furthermore, the rapid recovery following return to $30^{\circ} \mathrm{C}$ means that cumulative effects during this period are not found, so that no Effect 3 decrease is obtained.

Effect 4 involves the acceleration of sper-

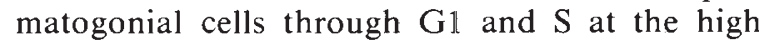
temperature, with a modification of their replication, or template preparation, so that more exchanges can form when these chromosomes subsequently undergo chiasma formation. This effect is recessive to the large Effect 3 decrease, but here, as no cumulative decrease of this type arose to cancel out the effect, a prolonged Effect 4 increase could be obtained, and might even have been maintained indefinitely.

The large reduction in chiasma frequency, with univalent production, which we have termed Effect 3 is of widespread occurrence, and has been shown to be produced by heat-treatments in a wide variety of plants, including many of economic importance. The Effect 4 increase, on the other hand, has yet to be demonstrated by others. So far we are the first to have studied it in detail. But we are confident that it will be found to be as widespread as the Effect 3 reduction if only others are prepared to look for it. It has usually been missed by previous workers because it is not usually produced at a constant high temperature. It is best shown after shorter heat-treatments of a few days at the high temperature followed by a return to a lower, control temperature. In our experience it is very reliably induced in a range of species, including some which do not show any Effect 3 reduction with high temperature treatment, such as the American grasshopper Melanoplus (Henderson, unpublished).

The relatively steady Effect 4 increase which we have found with regularly alternating heattreatments could, in theory, be of practical application to those plant breeders interested in increasing genetic recombination to aid the production of new varieties by conventional selection and hybridisation techniques. If successful on the species in which they are interested, the use of an alternating heat-treatment regime of this type might well increase recombination frequencies with less deleterious side effects than would be provided by some of the other agencies used, such as $\mathrm{X}$-rays or mutagens. 


\section{REFERENCES}

BUSS, M. E. 1971. The Experimental Control of Chromosome Pairing and Chiasma Formation. Ph.D. Thesis, University of Cambridge.

BUSS, M. F. AND HENDERSON, S. A. 1988. The effects of elevated temperature on chiasma formation in Locusta migratoria. Chromosoma (in press).

HENDERSON, S. A. 1966. The time of chiasma formation in relation to the time of DNA synthesis. Nature 211, 10431047.
HENDERSON, S. A. 1970. The time and place of meiotic crossingover. Ann. Rev. Genet. 4, 295-324.

HENDTRSON, S. A. 1988 a. Four effects of elevated temperature on chiasma formation in the locust Schistocerca gregaria. Heredity, 60, 387-401.

HENDERSON, S. A. 1988 b. Interactions between heat-induced chiasma frequency changes in Schistocerca gregaria. Heredity, 62, 45-49. 